This is the final peer-reviewed accepted manuscript of:

B. Lazzari; R. Nibbi - Energy decay in Green-Naghdi thermoelasticity with diffusion and dissipative boundary controls -

Journal of Thermal Stresses, vol. 40 (2017), pp. 917-927.

The final published version is available online at: http://dx.doi.org/10.1080/01495739.2016.1261262

Rights / License:

The terms and conditions for the reuse of this version of the manuscript are specified in the publishing policy. For all terms of use and more information see the publisher's website. 


\title{
Energy decay in Green-Naghdi thermoelasticity with diffusion and dissipative boundary controls
}

\author{
Barbara Lazzari Roberta Nibbi
}

\begin{abstract}
We study the asymptotic behavior of the solutions of a 3-D hyperbolic system arising in the Green-Naghdi models of thermoelasticity with diffusion of type II and III, in presence of dissipative boundary controls and prove that the energy exponentially decays in time.
\end{abstract}

\section{Introduction}

Diffusion in solids is an important topic in industrial applications, in fact diffusion processes play a key role in the kinetics of many micro-structural changes that occur during processing of metals, alloys, ceramics, semi-conductors, glasses, and polymers. Moreover thermodiffusion processes have a crucial role in aero-spatial and energetic engineering when studying, for example, the reentry from the space of vehicles and processes of extraction of oil.

Thermodiffusion in an elastic solid is due to the coupling of the fields of temperature, 
mass diffusion and that of strain. Nowacki $[1,2]$ developed the theory of thermoelastic diffusion in the context of the coupled thermoelastic model making use of the Fourier and Fick Laws for the heat and mass diffusion fluxes respectively. A brief survey on this theory and its applications can be found in [3]. Subsequently, Sherief et al. in [4] have proposed a theory which resolves the paradox of infinite propagation speeds. In this new theory the Fourier and Fick Laws are replaced by a Cattaneo-Maxwell type law (see e.g. [5]), which allows finite speeds of propagation of both thermal and diffusion waves. In the context of this generalized thermoelastic diffusion theory, various important problems have been considered (see for example [6, 7, 8, 9, 10] and references therein). Other interesting results have been obtained considering the effects of the CattaneoMaxwell type diffusion within the Green Nagdhi generalized thermoelasticity [11, 12].

Recently, another theory of thermoelastic diffusion with finite speeds of propagation has been presented in [13]. Here both the constitutive laws for heat and diffusing mass fluxes obey to Green-Naghdi type laws $[14,15]^{1}$.

The Green-Naghdi model of type II for thermoelasticity with diffusion leads to a conservative system. In this paper, following the approach proposed in $[16,17]$, we deal with the asymptotic behavior of solution of the thermoelastic diffusion system

\footnotetext{
${ }^{1}$ Green and Naghdi developed a thermo-mechanical theory of deformable continua that relies on an entropy balance law rather than an entropy inequality, where the heat conduction does not agree with the usual one. They proposed the use of the thermal displacement

$$
\alpha(\mathbf{x}, t)=\int_{t_{0}}^{t} \theta(\mathbf{x}, s) d s+\alpha_{0}
$$

where $\theta$ is the empirical temperature, and considered three theories labelled as type I, II and III, respectively. The type I thermoelasticity coincides with the classical one; in type II, the heat is allowed to propagate by means of thermal waves but without dissipating energy and, for this reason, it is also known as thermoelasticity without energy dissipation. The type III thermoelasticity includes the two above-mentioned theories as particular cases.
} 
in presence of mechanical and thermal dissipative boundary controls (see, for example, [18]), via semigroup theory. Within this approach, we don't need an additional damping

for the diffusion on the boundary to obtain the exponential decay of the energy. Instead, the Green-Naghdi model of type III, introducing internal thermo-diffusive dissipation effects, allows to get exponential stability in presence of mechanical dissipative boundary controls only.

The outline of the paper is the following. First we recall the basic equations of the linearized theory of thermoelastic diffusion without energy dissipation derived in [13] and prove existence, uniqueness and regularity of solutions for the relative initial boundary value problem. Then, we develop the needed estimates and prove the exponential decay of the energy. Finally, we extend the previous results to the type III model and propose some generalizations of our results.

\section{Setup of the problem}

We consider a linear homogeneous anisotropic thermoelastic diffusion solid in a bounded open set $\Omega$ of $\mathcal{R}^{3}$ with regular boundary $\partial \Omega$ such that

$$
\partial \Omega=\partial \Omega_{1} \cup \partial \Omega_{2}, \quad \overline{\partial \Omega_{1}} \cap \overline{\partial \Omega_{2}}=\text { ptyset, meas }\left\{\partial \Omega_{2}\right\} \neq 0
$$

where

$$
\begin{aligned}
& \partial \Omega_{1}=\left\{x \in \partial \Omega ;\left(x-x_{0}\right) \cdot n(x) \geq d>0\right\} \\
& \partial \Omega_{2}=\left\{x \in \partial \Omega ;\left(x-x_{0}\right) \cdot n(x)<0\right\}
\end{aligned}
$$


for a suitable $x_{0} \in \mathcal{R}^{3}$ and a given constant $d$.

The requirement (1) implies that $\Omega$ be strongly star shaped with respect to $x_{0}$.

The dynamic problem is described through the balance equations of the linear momentum, the entropy and the mass in the context of the linearized theory

$$
\begin{aligned}
\rho \ddot{\mathbf{u}}(\mathbf{x}, t) & =\nabla \cdot \mathbf{T}(\mathbf{x}, t)+\rho f(\mathbf{x}, t) \\
\rho \dot{S}(\mathbf{x}, t) & =-\nabla \cdot \mathbf{q}(\mathbf{x}, t)+\rho s(\mathbf{x}, t) \\
\dot{C}(\mathbf{x}, t) & =-\nabla \cdot \boldsymbol{\eta}(\mathbf{x}, t)
\end{aligned}
$$

where $\rho>0$ is the (constant) mass density, $\mathbf{u}$ it the displacement vector, $\mathbf{T}$ is the stress tensor, $\mathbf{f}$ is the body force per unit mass, $S$ is the entropy per unit mass, $\mathbf{q}$ is the heat flux, $s$ is the external heat source density, $C$ is the concentration of the diffusive material in the elastic body and $\boldsymbol{\eta}$ denotes the flow of the diffusing mass vector.

In this linear theory of Green Nagdhi type [14], we introduce the thermal displacement $\tau$ whose derivative coincides with the temperature $\theta$ and, in a similar way, the scalar function $\wp$ related to the chemical potential by the equation $\dot{\wp}=P$.

For this model, the compatibility with the Principles of Thermodynamics leads to 
the following constitutive equations (see [13])

$$
\begin{aligned}
\mathbf{T} & =\mathbb{A} \nabla \mathbf{u}+\mathbf{B} \theta+\mathbf{C} P \\
\rho S & =-\mathbf{B} \cdot \nabla \mathbf{u}+c \theta+\kappa P \\
\mathbf{q} & =-\mathbf{K} \nabla \tau-\mathbf{D} \nabla \wp \\
C & =-\mathbf{C} \cdot \nabla \mathbf{u}+\kappa \theta+m P \\
\boldsymbol{\eta} & =-\mathbf{H} \nabla \wp-\mathbf{D} \nabla \tau
\end{aligned}
$$

where $\mathbb{A}$ is a constant fourth order symmetric tensor positive definite, $\mathbf{B}, \mathbf{C}, \mathbf{D}, \mathbf{H}$ and $\mathbf{K}$ are constant second order symmetric tensors, $c, \kappa$ and $m$ are constants, such that there exist two positive constants $c_{1}$ and $c_{2}$ for which

$$
\begin{aligned}
& \mathbf{K} \nabla \tau \cdot \nabla \tau+2 \mathbf{D} \nabla \tau \cdot \nabla \wp+\mathbf{H} \nabla \wp \cdot \nabla \wp \geq c_{1}\left[|\nabla \tau|^{2}+|\nabla \wp|^{2}\right] \\
& c \theta^{2}+2 \kappa P \theta+m P^{2} \geq c_{2}\left(\theta^{2}+P^{2}\right)
\end{aligned}
$$

Moreover, as coupling is considered, the tensors B, C, D and the constant $\kappa$ are assumed to be non vanishing and, apart from (4), no further hypothesis needs to be taken into account.

By introducing the constitutive equations (3) into the balance equations (2), we obtain the following evolutive equations for the theory of thermoelastic diffusion materials 
without energy dissipation (i.e. Green Nagdhi type II)

$$
\begin{aligned}
& \rho \ddot{\mathbf{u}}(\mathbf{x}, t)= \nabla \cdot[\mathbb{A} \nabla \mathbf{u}(\mathbf{x}, t)+\mathbf{B} \theta(\mathbf{x}, t)+\mathbf{C} P(\mathbf{x}, t)]+\rho \mathbf{f}(\mathbf{x}, t) \\
& c \ddot{\tau}(\mathbf{x}, t)= \mathbf{B} \cdot \nabla \dot{\mathbf{u}}(\mathbf{x}, t)+\nabla \cdot[\mathbf{K} \nabla \tau(\mathbf{x}, t)+\mathbf{D} \nabla \wp(\mathbf{x}, t)] \\
&-\kappa \ddot{\wp}(\mathbf{x}, t)+\rho s(\mathbf{x}, t) \\
& m \ddot{\wp}(\mathbf{x}, t)=\mathbf{C} \cdot \nabla \dot{\mathbf{u}}(\mathbf{x}, t)+\nabla \cdot[\mathbf{H} \nabla \wp(\mathbf{x}, t)+\mathbf{D} \nabla \tau(\mathbf{x}, t)]-\kappa \ddot{\tau}(\mathbf{x}, t)
\end{aligned}
$$

As for the boundary and initial conditions we assume

$$
\begin{array}{ccc}
\mathbf{T}(\mathbf{x}, t) \mathbf{n}(\mathbf{x})=-\lambda \dot{\mathbf{u}}(\mathbf{x}, t), \quad \mathbf{q}(\mathbf{x}, t) \cdot \mathbf{n}(\mathbf{x})=\mu \dot{\tau}(\mathbf{x}, t) & \\
\mathbf{\eta}(\mathbf{x}, t) \cdot \mathbf{n}(\mathbf{x})=0 & \\
\mathbf{u}(\mathbf{x}, t)=0, \quad \tau(\mathbf{x}, t)=0, \quad \wp(\mathbf{x}, t)=0 & \mathbf{x} \in \partial \Omega_{1}
\end{array}
$$

with $\lambda$ and $\mu$ non-negative constants,

$$
\begin{array}{lll}
\mathbf{u}(\mathbf{x}, 0)=\mathbf{u}_{0}(\mathbf{x}), & \tau(\mathbf{x}, 0)=\tau_{0}(\mathbf{x}), & \wp(\mathbf{x}, 0)=\wp_{0}(\mathbf{x}) \\
\mathbf{u}(\mathbf{x}, 0)=\mathbf{v}_{0}(\mathbf{x}), & \dot{\tau}(\mathbf{x}, 0)=\dot{\theta}_{0}(\mathbf{x}), & \dot{\wp}(\mathbf{x}, 0)=P_{0}(\mathbf{x})
\end{array}
$$

From now on we shall drop the $\mathbf{x}$ variable whenever no ambiguity arises. In the sequel, we shall refer to problem $(5)-(7)$ as to problem $(\mathcal{P})$.

\section{Well-posedness}

In this section we shall prove the well-posedness of problem $(\mathcal{P})$ via semigroup theory. To this aim we transform the initial-boundary-value $(\mathcal{P})$ into a first order abstract 
Cauchy problem, following the formalism adopted in [13] for the same problem in presence of homogeneous Dirichlet boundary conditions (i.e. when $\partial \Omega_{1}=$ ptyset).

By introducing $\mathcal{U}=(\mathbf{u}, \mathbf{v}, \tau, \theta, \wp, P)$, problem $(\mathcal{P})$ can be rewritten as follows

$$
\dot{\mathcal{U}}(t)=\mathcal{A} \mathcal{U}(t)+\mathcal{F}(t), \quad \mathcal{U}(0)=\mathcal{U}_{0}
$$

where $\mathcal{U}_{0}$ is the initial data set, $\mathcal{F}$ is related to external sources, while the operator $\mathcal{A}$ is defined through the differential system (5). The technical procedure to define $\mathcal{A}$, and consequently $\mathcal{F}$, can be found in [13].

The natural setting in which to look for existence and uniqueness of solutions is the phadmissible states space $\mathcal{K}$, consisting in those functions $\mathcal{U}$ for which the total energy related to the problem $(\mathcal{P})$

$$
\begin{aligned}
\mathcal{E}= & \frac{1}{2} \int_{\Omega}\left[\rho|\mathbf{v}|^{2}+c|\theta|^{2}+2 \kappa \theta P+m P^{2}\right] d \mathbf{x} \\
& +\frac{1}{2} \int_{\Omega}\left[\mathbb{A} \nabla \mathbf{u} \cdot \nabla \mathbf{u}+\mathbf{K} \nabla \tau^{\cdot} \nabla \tau+2 \mathbf{D} \nabla \tau \cdot \nabla \wp+\mathbf{H} \nabla \wp \cdot \nabla \wp\right] d \mathbf{x}
\end{aligned}
$$

is well defined.

We observe that, as a consequence of the assumptions (4), the integral

$$
\int_{\Omega}\left[\rho|\mathbf{v}|^{2}+c|\theta|^{2}+2 \kappa \theta P+m|P|^{2}\right] d \mathbf{x}
$$

defines a norm that is equivalent to the usual norm in $\mathbf{L}^{2}(\Omega) \times L^{2}(\Omega) \times L^{2}(\Omega)$, where 
$\mathbf{L}^{2}(\Omega)=\left[L^{2}(\Omega)\right]^{3} ;$ moreover, thanks to the first Korn and the Poincaré inequalities,

$$
\int_{\Omega}[\mathbb{A} \nabla \mathbf{u} \cdot \nabla \mathbf{u}+\mathbf{K} \nabla \tau \cdot \nabla \tau+2 \mathbf{D} \nabla \tau \cdot \nabla \wp+\mathbf{H} \nabla \wp \cdot \nabla \wp] d \mathbf{x}
$$

defines a norm that is equivalent to the usual norm in $\mathbf{H}_{0_{2}}^{1}(\Omega) \times H_{0_{2}}^{1}(\Omega) \times H_{0_{2}}^{1}(\Omega)$, where $H_{0_{2}}^{1}(\Omega)=\left\{f \in H^{1}(\Omega) ; f=0\right.$ on $\left.\partial \Omega_{2}\right\}$ and $\mathbf{H}_{0_{2}}^{1}(\Omega)=\left[H_{0_{2}}^{1}(\Omega)\right]^{3}$. We can therefore conclude that

$$
\mathcal{K}=\mathbf{L}^{2}(\Omega) \times L^{2}(\Omega) \times L^{2}(\Omega) \times \mathbf{H}_{0_{2}}^{1}(\Omega) \times H_{0_{2}}^{1}(\Omega) \times H_{0_{2}}^{1}(\Omega)
$$

and the natural inner product in $\mathcal{K}$, related to $(9)$ is

$$
\begin{aligned}
& \left\langle(\mathbf{u}, \mathbf{v}, \tau, \theta, \wp, P),\left(\mathbf{u}^{*}, \mathbf{v}^{*}, \tau^{*}, \theta^{*}, \wp^{*}, P^{*}\right)\right\rangle= \\
& \int_{\Omega}\left[\rho \mathbf{v} \cdot \mathbf{v}^{*}+c \theta \theta^{*}+\kappa\left(\theta P^{*}+\theta^{*} P\right)+m P P^{*}\right] d \mathbf{x}+\int_{\Omega} \mathbb{A} \nabla \mathbf{u} \cdot \nabla \mathbf{u}^{*} d \mathbf{x} \\
& \quad+\int_{\Omega}\left[\mathbf{K} \nabla \tau \cdot \nabla \tau^{*}+\mathbf{H} \nabla \wp \cdot \nabla \wp^{*}+\mathbf{D} \nabla \tau \cdot \nabla \wp^{*}+\mathbf{D} \nabla \tau^{*} \cdot \nabla \wp\right] d \mathbf{x}
\end{aligned}
$$

The domain of the operator $\mathcal{A}$ is the dense subset of $\mathcal{D}$ defined by

$$
\mathcal{D}(\mathcal{A})=\left\{\mathcal{U} \in \mathcal{K}: \mathcal{A U} \in \mathcal{K} \text { and boundary conditions (6) on } \partial \Omega_{1} \text { hold }\right\}
$$

It easy to show that for $\mathcal{U} \in \mathcal{D}(\mathcal{A})$

$$
\operatorname{Range}(\mathcal{I}-\mathcal{A})=\mathcal{K}
$$


and, if $\mathcal{U}$ is solution of (8), then

$$
<\mathcal{A} \mathcal{U}, \mathcal{U}>=-\int_{\partial \Omega_{1}}\left[\lambda|\mathbf{v}|^{2}+\mu|\theta|^{2}\right] d \mathbf{a} \leq 0
$$

Therefore, from the Lumer-Phillips corollary to the Hille-Yosida theorem [19], the operator $\mathcal{A}$ generates a semigroup of contraction in $\mathcal{K}$.

Finally, making use of the results obtained in [20], it is possible to state the following theorem establishing the well posedness of problem $(\mathcal{P})$ in $\Omega \times \mathbb{R}^{+}$.

Theorem 1 If $\mathcal{F} \in W_{\text {loc }}^{1, p}\left(\mathcal{R}^{+} ; L^{2}(\Omega)\right)$ and $\mathcal{U}_{0} \in \mathcal{D}(\mathcal{A})$, then the system (8) admits one and only one strict solution $\mathcal{U} \in \mathcal{C}^{1}\left(\mathcal{R}^{+} ; \mathcal{K}\right) \cap \mathcal{C}\left(\mathcal{R}^{+} ; \mathcal{D}(\mathcal{A})\right)$.

\section{Exponential decay}

In order to show that an exponential decay of the energy (9) occurs over time, we assume that

$\left(\mathrm{h}_{1}\right) \operatorname{meas}\left\{\partial \Omega_{i}\right\}>0, i=1,2$,

$\left(\mathrm{h}_{2}\right)$ the constants $\lambda$ and $\mu$ in (6) must be positive,

$\left(h_{3}\right)$ the tensor $\mathbf{C}^{2}$ is positive definite i.e. there exists $\gamma>0$ such that

$$
|\mathbf{C w}|^{2} \geq \gamma|\mathbf{w}|^{2}
$$

Condition (10) ensures a strong coupling between mechanical and diffusive effects.

The main result of this section is the following theorem. 
Theorem 2 Let $\mathcal{U}$ be a solution of (8) with initial data $\mathbb{U}_{0} \in \mathcal{D}(\mathcal{A})$ and null sources. If the conditions $\left(\mathrm{h}_{1}\right),\left(\mathrm{h}_{2}\right)$ and $\left(\mathrm{h}_{3}\right)$ hold, then

$$
\mathcal{E}(t) \leq \delta_{2} e^{-\delta_{1} t} \mathcal{E}(0)
$$

where $\delta_{1}$ and $\delta_{2}$ are two positive constants.

In order to prove the above theorem, following [18], we introduce the functional

$$
\mathcal{L}_{t_{0}}(t)=\left(t+t_{0}\right) \mathcal{E}(t)+\mathcal{G}(t)
$$

with

$$
\begin{aligned}
\mathcal{G}(t)= & \rho \int_{\Omega} \mathbf{v}(t) \cdot[\nabla \mathbf{u}(t) \boldsymbol{\ell}+\mathbf{u}(t)] d \mathbf{x} \\
& +\int_{\Omega}[c \theta(t)-\mathbf{B} \cdot \nabla \mathbf{u}(t)+\kappa P(t)][\nabla \tau(t) \cdot \boldsymbol{\ell}+\tau(t)] d \mathbf{x} \\
& +\int_{\Omega}[m P(t)-\mathbf{C} \cdot \nabla \mathbf{u}(t)+\kappa \theta(t)][\nabla \wp \cdot \boldsymbol{\ell}+\wp] d \mathbf{x}
\end{aligned}
$$

where $\boldsymbol{\ell}(x)=\left(\mathbf{x}-\mathbf{x}_{0}\right)$, and prove the following key result

Lemma 1 Under the hypotheses of Theorem 2, the following inequality holds

$$
\frac{d}{d t}\left[\mathcal{L}_{t_{0}}(t)+\frac{1}{2} \int_{\partial \Omega_{1}}\left[\lambda|\mathbf{u}(t)|^{2}+\mu|\tau(t)|^{2}\right] d \mathbf{a}\right] \leq 0
$$

for $t_{0}$ be sufficiently large; moreover there exists a positive constant $\beta$, such that

$$
\mathcal{L}_{t_{0}}(T)-\mathcal{L}_{t_{0}}(0) \geq\left(T+t_{0}-\beta\right) \mathcal{E}(T)-\left(t_{0}+\beta\right) \mathcal{E}(0)
$$


for $T>0$.

Proof. Since the time derivative of $\mathcal{L}$ is

$$
\dot{\mathcal{L}}_{t_{0}}(t)=\left(t+t_{0}\right) \dot{\mathcal{E}}(t)+\mathcal{E}(t)+\dot{\mathcal{G}}(t)
$$

we need to estimate $\dot{\mathcal{E}}$ and $\dot{\mathcal{G}}$ when $\mathcal{U}$ is a solution of (8).

Applying the divergence theorem, recalling the symmetry of the constitutive tensors and the boundary conditions (6), it is easy to show that

$$
\begin{aligned}
\dot{\mathcal{E}}(t) & =\int_{\partial \Omega_{1}}[\mathbf{T}(t) \mathbf{n} \cdot \mathbf{v}(t)-\mathbf{q}(t) \cdot \mathbf{n} \theta(t)-\boldsymbol{\eta} \cdot \mathbf{n} P] d \mathbf{a} \\
& =-\int_{\partial \Omega_{1}}\left[\lambda|\mathbf{v}|^{2}+\mu|\theta|^{2}\right] d \mathbf{a} \leq 0
\end{aligned}
$$


while

$$
\begin{aligned}
\dot{\mathcal{G}}(t)= & -\mathcal{E}(t)+\frac{1}{2} \int_{\partial \Omega_{1}}\left[\rho|v(t)|^{2}+c \theta^{2}(t)+2 \kappa \theta(t) P(t)+m P^{2}(t)\right] \boldsymbol{\ell} \cdot \mathbf{n} d \mathbf{a} \\
& +\int_{\partial \Omega_{1}}\left[\mathbf{T}(t) \mathbf{n} \cdot(\nabla \mathbf{u}(t) \boldsymbol{\ell}+\mathbf{u}(t))-\frac{1}{2} \mathbb{A} \nabla \mathbf{u}(t) \cdot \nabla \mathbf{u}(t) \boldsymbol{\ell} \cdot \mathbf{n}\right] d \mathbf{a} \\
& -\int_{\partial \Omega_{1}}\left[\mathbf{q}(t) \cdot \mathbf{n}(\nabla \tau(t) \cdot \boldsymbol{\ell}+\tau(t))-\frac{1}{2} \mathbf{q}(t) \cdot \nabla \tau(t) \boldsymbol{\ell} \cdot \mathbf{n}\right] d \mathbf{a} \\
& +\int_{\partial \Omega_{1}} \boldsymbol{\eta}(t) \cdot \nabla \wp(t) \boldsymbol{\ell} \cdot \mathbf{n} d \mathbf{a} \\
& +\int_{\partial \Omega_{2}}\left[\mathbb{A} \nabla \mathbf{u}(t) \mathbf{n} \cdot(\nabla \mathbf{u}(t) \boldsymbol{\ell})-\frac{1}{2} \mathbb{A} \nabla \mathbf{u}(t) \cdot \nabla \mathbf{u}(t) \boldsymbol{\ell} \cdot \mathbf{n}\right] d \mathbf{a} \\
& -\int_{\partial \Omega_{2}}\left[\mathbf{q}(t) \cdot \mathbf{n}(\nabla \tau(t) \cdot \boldsymbol{\ell})-\frac{1}{2} \mathbf{q}(t) \cdot \nabla \tau(t) \boldsymbol{\ell} \cdot \mathbf{n}\right] d \mathbf{a} \\
& -\int_{\partial \Omega_{2}}\left[\boldsymbol{\eta}(t) \cdot \mathbf{n}(\nabla \wp(t) \cdot \boldsymbol{\ell})-\frac{1}{2} \boldsymbol{\eta}(t) \cdot \nabla \wp(t) \boldsymbol{\ell} \cdot \mathbf{n}\right] d \mathbf{a} \\
= & -\mathcal{E}(t)+I_{\partial \Omega_{1}}(t)+I_{\partial \Omega_{2}}(t)
\end{aligned}
$$

Since it is known that the condition $w_{\mid \partial \Omega_{2}}=0$ implies $\nabla w=(\nabla w \cdot \mathbf{n}) \mathbf{n}$ on $\partial \Omega_{2}$, then

$$
\begin{gathered}
I_{\partial \Omega_{2}}(t)=\frac{1}{2} \int_{\partial \Omega_{2}}[\mathbb{A} \nabla \mathbf{u} \cdot \nabla \mathbf{u}-\mathbf{q} \cdot \nabla \tau-\boldsymbol{\eta} \cdot \nabla \wp] \boldsymbol{\ell} \cdot \mathbf{n} d \mathbf{a} \\
=\frac{1}{2} \int_{\partial \Omega_{2}}[\mathbb{A} \nabla \mathbf{u} \cdot \nabla \mathbf{u}+\mathbf{K} \nabla \tau \cdot \nabla \tau+2 \mathbf{D} \nabla \tau \cdot \nabla \wp+\mathbf{H} \nabla \wp \cdot \nabla \wp] \boldsymbol{\ell} \cdot \mathbf{n} d \mathbf{a} \leq 0
\end{gathered}
$$

in virtue of the assumptions on the constitutive coefficients and $(1)_{2}$.

We now proceed to estimate the various terms of $I_{\partial \Omega_{1}}$. 
Preliminarily, we provide an estimate for $P^{2}$. By virtue of $(3)_{1}$ and (10), we have

$$
\begin{aligned}
|P(t)|^{2} & \leq \frac{1}{\gamma}|P(t) \mathbf{C n}|^{2} \leq \frac{2}{\gamma}\left[|\mathbf{T}(t) \mathbf{n}|^{2}+|\mathbb{A} \nabla \mathbf{u}(t) \mathbf{n}-\theta(t) \mathbf{B n}|^{2}\right] \\
& \leq \frac{2}{\gamma}\left[|\mathbf{T}(t) \mathbf{n}|^{2}+\frac{\gamma}{4(m+|k|)} \mathbb{A} \nabla \mathbf{u}(t) \cdot \nabla \mathbf{u}(t)+\alpha_{1}|\theta(t)|^{2}\right]
\end{aligned}
$$

where, in the last inequality, we have used the positive definiteness of $\mathbb{A}$ and classical AGM-inequalities with $\alpha_{1}$ suitable positive constant. Moreover from the hypotheses on the constitutive equations and $(1)_{1}$ we get

$$
\begin{aligned}
\int_{\partial \Omega_{1}} & {[\mathbf{q}(t) \cdot \nabla \tau(t)+\boldsymbol{\eta}(t) \cdot \nabla \wp(t)] \boldsymbol{\ell} \cdot \mathbf{n} d \mathbf{a} } \\
= & -\int_{\partial \Omega_{1}}[\mathbf{K}(t) \nabla \tau(t) \cdot \nabla \tau(t)+\mathbf{H}(t) \nabla \wp(t) \cdot \nabla \wp(t)] \boldsymbol{\ell} \cdot \mathbf{n} d \mathbf{a} \\
& -2 \int_{\partial \Omega_{1}} \mathbf{D}(t) \nabla \tau(t) \cdot \nabla \wp(t) \boldsymbol{\ell} \cdot \mathbf{n} d \mathbf{a} \\
\leq & -c_{1} \int_{\partial \Omega_{1}}\left[|\nabla \tau(t)|^{2}+|\nabla \wp(t)|^{2}\right] \boldsymbol{\ell} \cdot \mathbf{n} d \mathbf{a} \\
\leq & -c_{1} d \int_{\partial \Omega_{1}}\left[|\nabla \tau(t)|^{2}+|\nabla \wp(t)|^{2}\right] d \mathbf{a}
\end{aligned}
$$

As a consequence of (16) we obtain

$$
\begin{aligned}
& \int_{\partial \Omega_{1}}\left[\rho|v(t)|^{2}+c|\theta(t)|^{2}+2 \kappa \theta(t) P(t)+m|P(t)|^{2}\right] \boldsymbol{\ell} \cdot \mathbf{n} d \mathbf{a} \\
& \quad \leq \int_{\partial \Omega_{1}}\left[\rho|v(t)|^{2}+(c+|\kappa|)|\theta(t)|^{2}+(m+|\kappa|) P^{2}(t)\right] \boldsymbol{\ell} \cdot \mathbf{n} d \mathbf{a} \\
& \quad \leq \int_{\partial \Omega_{1}}\left[\rho|v(t)|^{2}+\alpha_{2}|\mathbf{T}(t) \mathbf{n}|^{2}+\alpha_{3}|\theta(t)|^{2}+\frac{1}{2} \mathbb{A} \nabla \mathbf{u}(t) \cdot \nabla \mathbf{u}(t)\right] \boldsymbol{\ell} \cdot \mathbf{n} d \mathbf{a}
\end{aligned}
$$

with $\alpha_{2}$ and $\alpha_{3}$ positive constants. 
Through the same arguments of the previous inequality and taking into account the boundary conditions (6) we have

$$
\begin{gathered}
\int_{\partial \Omega_{1}}\left[\mathbf{T}(t) \mathbf{n} \cdot(\nabla \mathbf{u}(t) \boldsymbol{\ell}+\mathbf{u}(t))-\frac{1}{2} \mathbb{A} \nabla \mathbf{u}(t) \cdot \nabla \mathbf{u}(t) \boldsymbol{\ell} \cdot \mathbf{n}\right] d \mathbf{a} \\
\leq \int_{\partial \Omega_{1}}\left[\alpha_{4}|\mathbf{T}(t) \mathbf{n}|^{2}-\frac{\lambda}{2} \frac{d}{d t}|\mathbf{u}(t)|^{2}-\frac{1}{4} \mathbb{A} \nabla \mathbf{u}(t) \cdot \nabla \mathbf{u}(t) \boldsymbol{\ell} \cdot \mathbf{n}\right] d \mathbf{a} \\
-\int_{\partial \Omega_{1}} \mathbf{q}(t) \cdot \mathbf{n}[\nabla \tau(t) \cdot \boldsymbol{\ell}+\tau(t)] d \mathbf{a} \\
\leq \frac{1}{2} \int_{\partial \Omega_{1}}\left[\alpha_{5}|\mathbf{q}(t) \cdot \mathbf{n}|^{2}-\mu \frac{d}{d t}|\tau(t)|^{2}+c_{1} d|\nabla \tau(t)|^{2}\right] d \mathbf{a}
\end{gathered}
$$

with $\alpha_{4}$ and $\alpha_{5}$ positive constants depending on $d$, on the diameter of $\Omega$ and on the positive coerciveness coefficients of $\mathbb{A}$ and $c_{1}$.

The inequalities (15)-(20) allow to evaluate $\dot{\mathcal{G}}$ as follows

$$
\begin{aligned}
\dot{\mathcal{G}}(t) \leq-\mathcal{E}(t)+I_{\partial \Omega_{1}}(t) \leq & -\mathcal{E}(t)+\int_{\partial \Omega_{1}}\left[\alpha_{6}|\mathbf{v}(t)|^{2}+\alpha_{7}|\theta(t)|^{2}\right] d a \\
& -\frac{1}{2} \frac{d}{d t} \int_{\partial \Omega_{1}}\left[\lambda|\mathbf{u}(t)|^{2}+\mu|\tau(t)|^{2}\right] d \mathbf{a}
\end{aligned}
$$

with $\alpha_{6}$ and $\alpha_{7}$ suitable positive constants. Therefore, substituting (14) and (21) into (13), we obtain

$$
\begin{aligned}
& \frac{d}{d t}\left[\mathcal{L}_{t_{0}}(t)+\frac{1}{2} \int_{\partial \Omega_{1}}\left[\lambda|\mathbf{u}(t)|^{2}+\mu|\tau(t)|^{2}\right] d \mathbf{a}\right] \\
& \leq\left[\alpha_{6}-\lambda\left(t+t_{0}\right)\right] \int_{\partial \Omega_{1}}|\mathbf{v}(t)|^{2} d \mathbf{a}+\left[\alpha_{7}-\mu\left(t+t_{0}\right)\right] \int_{\partial \Omega_{1}}|\theta(t)|^{2} d \mathbf{a}
\end{aligned}
$$

By choosing $t_{0}$ sufficiently large we get (11). 
Since

$$
\left(t+t_{0}\right) \mathcal{E}(t)-|\mathcal{G}(t)| \leq \mathcal{L}_{t_{0}}(t) \leq\left(t+t_{0}\right) \mathcal{E}(t)+|\mathcal{G}(t)|
$$

to prove (12), it is sufficent to estimate $|\mathcal{G}|$. Using the Poincaré inequality, together with the hypotheses of positive definiteness of the constitutive coefficients, we have

$$
\begin{aligned}
|\mathcal{G}(t)| \leq & \left|\rho \int_{\Omega} \mathbf{v}(t) \cdot[\nabla \mathbf{u}(t) \boldsymbol{\ell}+\mathbf{u}(t)] d \mathbf{x}\right| \\
& +\left|\int_{\Omega}[c \theta(t)-\mathbf{B} \cdot \nabla \mathbf{u}(t)++\kappa P(t)][\nabla \tau(t) \cdot \boldsymbol{\ell}+\tau(t)] d \mathbf{x}\right| \\
& +\left|\int_{\Omega}[c \theta(t)-\mathbf{B} \cdot \nabla \mathbf{u}(t)++\kappa P(t)][\nabla \tau(t) \cdot \boldsymbol{\ell}+\tau(t)] d \mathbf{x}\right| \\
\leq & \frac{\beta_{1}}{2} \int_{\Omega}\left[\rho|\mathbf{v}(t)|^{2}+\frac{1}{2} \mathbb{A} \nabla \mathbf{u}(t) \cdot \nabla \mathbf{u}(t)\right] d \mathbf{x} \\
& +\frac{\beta_{2}}{2} \int_{\Omega}\left[c|\theta(t)|^{2}+2 \kappa \theta(t) P(t)+m|P(t)|^{2}+\frac{1}{2} \mathbb{A} \nabla \mathbf{u}(t) \cdot \nabla \mathbf{u}(t)\right] d \mathbf{x} \\
& +\frac{\beta_{2}}{2} \int_{\Omega}[\mathbf{K}(t) \nabla \tau(t) \cdot \nabla \tau(t)+\mathbf{H}(t) \nabla \wp(t) \cdot \nabla \wp(t)] d \mathbf{x}
\end{aligned}
$$

Letting $\beta=\beta_{1}+\beta_{2}$, we obtain (12).

We are now able to proceed to prove the main result of this section.

Proof of Theorem 2 Taking $t_{0}$ sufficiently large and integrating (11) from 0 to $T$ we get

$$
\mathcal{L}_{t_{0}}(T)-\mathcal{L}_{t_{0}}(0) \leq-\frac{1}{2} \int_{\Omega_{1}}\left[\lambda|\mathbf{u}(T)|^{2}+\mu|\tau(T)|^{2}\right] d \mathbf{a}+h_{1} \leq h_{1}
$$

where

$$
h_{1}=\frac{1}{2} \int_{\partial \Omega_{1}}\left[\lambda|\mathbf{u}(0)|^{2}+\mu|\tau(0)|^{2}\right] d \mathbf{a}
$$


Finally, from the comparison of (12) and (22), it follows that

$$
\mathcal{E}(T) \leq \frac{\left(t_{0}+\beta\right) \mathcal{E}(0)+h_{1}}{T+t_{0}-\beta}
$$

Estimate (23) ensures the exponential decay of $\mathcal{E}$ thanks to the semigroup properties proved in the preceding section (see, for instance,[19, Theorem 4.1]).

\section{Some results on the type III model}

Here we extend the results of the previous section to the type III model, characterized by the following constitutive equations for the heat and diffusing mass flux vectors

$$
\begin{aligned}
& \mathbf{q}=-\mathbf{K} \nabla \tau-\mathbf{D} \nabla \wp-\widetilde{\mathbf{K}} \nabla \theta-\widetilde{\mathbf{D}} \nabla P \\
& \boldsymbol{\eta}=-\mathbf{H} \nabla \wp-\mathbf{D} \nabla \tau-\widetilde{\mathbf{H}} \nabla P-\widetilde{\mathbf{D}} \nabla \theta
\end{aligned}
$$

where $\widetilde{\mathbf{K}}, \widetilde{\mathbf{H}}$ and $\widetilde{\mathbf{D}}$ are second order symmetric tensors, which satisfy the following restriction

$$
\widetilde{\mathbf{K}} \nabla \theta \cdot \nabla \theta+2 \widetilde{\mathbf{D}} \nabla \theta \cdot \nabla P+\widetilde{\mathbf{H}} \nabla P \cdot \nabla P \geq c_{3}\left[|\nabla \theta|^{2}+|\nabla P|^{2}\right]
$$

with $c_{3}$ positive constant.

Taking into account the thermal and diffusive internal dissipative effects due to the constitutive equations (24), we are able to get exponential decay results also without the boundary dissipative thermal control. For this reason we here consider the following 
conditions on $\partial \Omega_{1}$

$$
\mathbf{T}(\mathbf{x}, t) \mathbf{n}(\mathbf{x})=-\lambda \dot{\mathbf{u}}(\mathbf{x}, t), \mathbf{q}(\mathbf{x}, t) \cdot \mathbf{n}(\mathbf{x})=\boldsymbol{\eta}(\mathbf{x}, t) \cdot \mathbf{n}(\mathbf{x})=0 \quad \mathbf{x} \in \partial \Omega_{1}
$$

together with the Dirichlet boundary conditions $(6)_{2}$ on $\partial \Omega_{2}$.

Using techniques similar to those employed in the previous section, it is possible to state that this new evolutive problem is well posed (see also [17]). In order to prove the exponential decay in time of the energy, we replace the auxiliary functional $\mathcal{L}_{t_{0}}$ with the following

$$
\mathcal{M}_{t_{0}}(t)=\left(t+t_{0}\right) \mathcal{E}(t)+\mathcal{H}(t)
$$

where

$$
\begin{aligned}
\mathcal{H}(t)= & 2 \rho \int_{\Omega} \mathbf{v}(t) \cdot[\nabla \mathbf{u}(t) \boldsymbol{\ell}+\mathbf{u}(t)] d \mathbf{x} \\
& +\int_{\Omega}[c \theta(t)-\mathbf{B} \cdot \nabla \mathbf{u}(t)+\kappa P(t)] \tau(t) d \mathbf{x} \\
& +\int_{\Omega}[m P(t)-\mathbf{C} \cdot \nabla \mathbf{u}(t)+\kappa \theta(t)] \wp d \mathbf{x}
\end{aligned}
$$

To evaluate its time derivative

$$
\dot{\mathcal{M}}_{t_{0}}(t)=\left(t+t_{0}\right) \dot{\mathcal{E}}(t)+\mathcal{E}(t)+\dot{\mathcal{H}}(t)
$$

we observe that from the boundary conditions (26)-(6) 2 and the restriction (25), we 
get

$$
\begin{aligned}
\dot{\mathcal{E}}(t)= & \int_{\partial \Omega_{1}} \mathbf{T}(t) \mathbf{n} \cdot \mathbf{v}(t) d \mathbf{a} \\
& -\int_{\Omega}[\widetilde{\mathbf{K}} \nabla \theta(t) \cdot \nabla \theta(t)+2 \widetilde{\mathbf{D}} \nabla \theta(t) \cdot \nabla P(t)+\widetilde{\mathbf{H}} \nabla P(t) \cdot \nabla P(t)] d \mathbf{x} \\
\leq & -\int_{\partial \Omega_{1}} \lambda|\mathbf{v}(t)|^{2} d \mathbf{a}-c_{3} \int_{\Omega}\left[|\nabla \theta(t)|^{2}+|\nabla P(t)|^{2}\right] d \mathbf{x} \leq 0
\end{aligned}
$$

while

$$
\begin{aligned}
\dot{\mathcal{H}}(t)= & -\mathcal{E}(t)-\frac{1}{2} \int_{\Omega}\left[\mathbb{A} \nabla \mathbf{u}(t) \cdot \nabla \mathbf{u}(t)+\rho|\mathbf{v}(t)|^{2}\right] d \mathbf{x} \\
& -\frac{1}{2} \int_{\Omega}[\mathbf{K} \nabla \tau(t) \cdot \nabla \tau(t)+2 \mathbf{D} \nabla \tau(t) \cdot \nabla \wp(t)+\mathbf{H} \nabla \wp(t) \cdot \nabla \wp(t)] d \mathbf{x} \\
& -\int_{\Omega}[\widetilde{\mathbf{K}} \nabla \theta(t) \cdot \nabla \tau(t)+\widetilde{\mathbf{H}} \nabla P(t) \cdot \nabla \wp(t)] d \mathbf{x} \\
& -\int_{\Omega}[\widetilde{\mathbf{D}} \nabla \theta(t) \cdot \nabla \wp(t)+\widetilde{\mathbf{D}} \nabla \tau(t) \cdot \nabla P(t)] d \mathbf{x} \\
& +\frac{3}{2} \int_{\Omega}\left[c|\theta(t)|^{2}+2 \kappa P(t) \theta(t)+m|P(t)|^{2}\right] d \mathbf{x} \\
& +\int_{\Omega} \mathbf{B} \cdot \nabla \mathbf{u}(t)[2 \nabla \theta(t) \cdot \boldsymbol{\ell}+\theta(t)] d \mathbf{x} \\
& +\int_{\Omega} \mathbf{C} \cdot \nabla \mathbf{u}(t)[2 \nabla P(t) \cdot \boldsymbol{\ell}+P(t)] d \mathbf{x} \\
& +\int_{\partial \Omega}[2 \mathbf{T}(t) \mathbf{n} \cdot(\nabla \mathbf{u}(t) \boldsymbol{\ell}+\mathbf{u}(t))-\mathbb{A} \nabla \mathbf{u}(t) \cdot \nabla \mathbf{u}(t) \boldsymbol{\ell} \cdot \mathbf{n}] d \mathbf{a} \\
& +\int_{\partial \Omega}\left[\rho|\mathbf{v}(t)|^{2}-2(\theta(t) \mathbf{B}+P(t) \mathbf{C}) \cdot \nabla \mathbf{u}(t)\right] \boldsymbol{\ell} \cdot \mathbf{n} d \mathbf{a} \\
= & -\mathcal{E}(t)+J_{\Omega}(t)+J_{\partial \Omega}(t)
\end{aligned}
$$


therefore

$$
\begin{aligned}
\dot{\mathcal{M}}_{t_{0}}(t) \leq & -\left(t+t_{0}\right)\left[\int_{\partial \Omega_{1}} \lambda|\mathbf{v}(t)|^{2} d \mathbf{a}-c_{3} \int_{\Omega}\left[|\nabla \theta(t)|^{2}+|\nabla P(t)|^{2}\right] d \mathbf{x}\right] \\
& +J_{\Omega}(t)+J_{\partial \Omega}(t)
\end{aligned}
$$

Making use of the classical AGM and Poincaré inequalities, we get the following estimate for $J_{\Omega}$

$$
J_{\Omega}(t) \leq c_{3} \delta_{1} \int_{\Omega}\left[|\nabla \theta(t)|^{2}+|\nabla P(t)|^{2}\right] d \mathbf{x}
$$

moreover, the application of the trace theorems to estimate $P$ and $\theta$ on $\partial \Omega$ in terms of $\nabla \theta$ and $\nabla P$ in $\Omega$ leads to

$$
\begin{aligned}
J_{\partial \Omega}(t) \leq & -\lambda \frac{d}{d t} \int_{\partial \Omega_{1}}|\mathbf{u}(t)|^{2} d \mathbf{a} \\
& +c_{3} \delta_{2} \int_{\Omega}\left[|\nabla \theta(t)|^{2}+|\nabla P(t)|^{2}\right] d \mathbf{x}+\lambda \delta_{3} \int_{\partial \Omega_{1}}|\mathbf{v}(t)|^{2} d \mathbf{a}
\end{aligned}
$$

As a consequence of the above inequalities, we can conclude that

$$
\begin{aligned}
\frac{d}{d t}\left[\mathcal{M}_{t_{0}}(t)\right. & \left.+\lambda \int_{\partial \Omega_{1}}|\mathbf{u}(t)|^{2} d \mathbf{a}\right] \leq-\lambda\left(t+t_{0}-\delta_{3}\right) \int_{\partial \Omega_{1}} \lambda|\mathbf{v}(t)|^{2} d \mathbf{a} \\
& -c_{3}\left(t+t_{0}-\delta_{1}-\delta_{2}\right) \int_{\Omega}\left[|\nabla \theta(t)|^{2}+|\nabla P(t)|^{2}\right] d \mathbf{x} \leq 0
\end{aligned}
$$

for $t_{0}>\delta_{1}+\delta_{2}+\delta_{3}$.

Finally, since also $\mathcal{M}_{t_{0}}$ satisfies (12), Theorem 2 ensures the exponential decay of the energy in this type III model. 


\section{Conclusion and discussion}

In this paper we have considered the Green Nagdhi model for thermoelasticity with diffusion, proposed in [13]. The absence of internal dissipation in the type II model suggests the introduction of suitable dissipative controls to obtains asymptotic decay results for the solutions of the evolutive problem.

Here we have restricted our attention to three-dimensional strongly star-shaped bounded domains and introduced particular linear boundary controls of viscous type $(6)_{1}$, to describe an energy absorbing surface. We underline that the boundary condition $\boldsymbol{\eta} \cdot \mathbf{n}=0$ is conservative, but the strong internal coupling (10) allows to get the exponential decay of the full thermo-diffusive-elastic energy (9).

Moreover, in the type III model, which exhibits thermal and diffusive internal dissipation, the exponential decay of the energy is obtained with only one mechanical dissipative control on the boundary.

It is worth to observe that these results can be easily generalized to boundary controls of memory type such as those proposed in [21]:

$$
\mathbf{T}(\mathbf{x}, t) \mathbf{n}(\mathbf{x})=-\lambda \dot{\mathbf{u}}(\mathbf{x}, t)+\int_{0}^{\infty} \nu(s)[\mathbf{u}(\mathbf{x}, t-s)-\mathbf{u}(\mathbf{x}, t)] d s
$$

Boundary conditions of this kind are dissipative under suitable restrictions on $\lambda$ and on the memory kernel $\nu$, but results about the exponential decay of the energy are obtained if and only if $\lambda$ is positive and $\nu$ exhibits an exponential decay behavior [22], i.e.

$$
\nu(s)<0, \quad \frac{d}{d s} \nu(s) \geq 0, \quad \frac{d}{d s} \nu(s)+k \nu(s) \geq 0, \quad s \geq 0
$$


with $k>0$.

Finally, we recall that the requirement meas $\left\{\partial \Omega_{2}\right\}>0$ can be eliminated (see for instance [18]). In the case meas $\left\{\partial \Omega_{2}\right\}=0$, we still get the exponential decay of the energy, while the solution $(\mathbf{u}, \tau, \wp)$ tends to a constant value equal to the mean value of the initial data $\left(\mathbf{u}_{0}, \tau_{0}, \wp_{0}\right)$.

\section{References}

[1] W. Nowacki, Dynamical problems of thermo diffusion in solids, I, II, III, Bull. Acad. Pol. Sci., Ser. Sci. Tech., vol. 22, pp. 55-64, 129-135, 257-266, 1974.

[2] W. Nowacki, Thermodiffusion in solid body, Mech. Teoret. Stos., vol. 13, pp. 143-158, 1975.

[3] Z. Olesiak, Problems of thermodiffusion of deformable solids, Mater. Sci., vol. 34, pp. 297-303, 1998.

[4] H. H. Sherief, F. A. Hamza, and H. A. Saleh, The theory of generalized thermoelastic diffusion, Internat. J. Engrg. Sci., vol. 42, pp. 591-608, 2004.

[5] H. W. Lord and Y. Shulman, A generalized dynamical theory of thermoelasticity, J. Mech. Phys. Solids, vol. 15, pp. 299-309, 1967.

[6] M. Aouadi, Uniqueness and reciprocity theorems in the theory of generalized thermoelastic diffusion, J. Thermal Stresses, vol. 30, pp. 665-678, 2007. 
[7] H. H. Sherief and H. A. Saleh, A half-space problem in the theory of generalized thermoelastic diffusion, Int. J. Solids Struc., vol. 42, pp. 4484-4493, 2005.

[8] B. Singh, Reflection of P and SV waves from free surface of an elastic solid with generalized thermodiffusion, J. Earth Syst. Sci., vol. 114(2):159-168, 2005.

[9] J. Tripathi, G. Kedar, and K. Deshmukh, Generalized thermoelastic diffusion problem in a thick circular plate with axisymmetric heat supply, Acta Mech., pages $1-14,2015$.

[10] R. Xia, X. Tian, and Y. Shen, The influence of diffusion on generalized thermoelastic problems of infinite body with a cylindrical cavity, Internat. J. Engrg. Sci., vol. 47(5):669-679, 2009.

[11] M. I. Othman, S. Y. Atwa, and R. Farouk, The effect of diffusion on twodimensional problem of generalized thermoelasticity with Green-Naghdi theory, Int. Commun. Heat Mass Transf., vol. 36, pp. 857-864, 2009.

[12] B. Singh, Wave propagation in a Green-Naghdi thermoelastic solid with diffusion, Int. J. Thermophysics, vol. 34(3):553-566, 2013.

[13] M. Aouadi, B. Lazzari, and R. Nibbi, A theory of thermoelasticity with diffusion under Green-Naghdi models, ZAMM Z. Angew. Math. Mech., vol. 94, pp. 837$852,2014$.

[14] A. E. Green and P. M. Naghdi, Thermoelasticity without energy dissipation, J. Elasticity, vol. 31, pp. 189-208, 1993. 
[15] A. E. Green and P. M. Naghdi, A unified procedure for construction of theories of deformable media: I. Classical continuum physics, II. Generalized continua, III. Mixtures of interacting continua, Proc. Roy. Soc. London A, vol. 448, pp. 335-356, 357-377, 379-388, 1995.

[16] M. Aouadi, B. Lazzari, and R. Nibbi, Energy decay in thermoelastic diffusion theory with second sound and dissipative boundary, Meccanica, vol. 48, pp. 2159-2171, 2013.

[17] B. Lazzari and R. Nibbi, On the exponential decay in thermoelasticity without energy dissipation and of type III in presence of an absorbing boundary, J. Math. Anal. Appl., vol. 338, pp. 317-329, 2008.

[18] J. Lagnese, Decay of solutions of wave equations in a bounded region with boundary dissipation, J. Diff. Equations, vol. 50, pp. 163-182, 1983.

[19] A. Pazy, Semigroups of linear operators and applications to partial differential equations, volume 44 of Applied Mathematical Sciences, Springer-Verlag, New York, 1983.

[20] G. Da Prato and E. Sinestrari, Differential operators with non dense domain, Ann. Scuola Norm. Sup. Pisa Cl. Sci., vol. 14, pp. 285-344, 1987.

[21] G. Propst and J. Prüss, On wave equations with boundary dissipation of memory type, J. Integral Equations Appl., vol. 8, pp. 99-123, 1996.

[22] C. A. Bosello, B. Lazzari, and R. Nibbi, A viscous boundary condition with memory in linear elasticity, Internat. J. Engrg. Sci., vol. 45, pp. 94-110, 2007. 